International Journal of Canadian Studies

Revue internationale d'études canadiennes

\title{
Les rêves américain et canadien des Jobin. Une famille bourgeoise de Québec aux États-Unis, 1890-1990
}

\section{Leslie Choquette}

Numéro 44, 2011

Americanity, Continentalism and Globalisation

Américanité, continentalité et mondialisation

URI : https://id.erudit.org/iderudit/1010083ar

DOI : https://doi.org/10.7202/1010083ar

Aller au sommaire du numéro

\section{Éditeur(s)}

Conseil international d'études canadiennes

\section{ISSN}

1180-3991 (imprimé)

1923-5291 (numérique)

Découvrir la revue

Citer cette note

Choquette, L. (2011). Les rêves américain et canadien des Jobin. Une famille bourgeoise de Québec aux États-Unis, 1890-1990. International Journal of Canadian Studies / Revue internationale d'études canadiennes, (44), 111-118. https://doi.org/10.7202/1010083ar

\section{Résumé de l'article}

Basée sur la Collection Jobin, un fonds familial franco-américain conservé à l'Institut français d'Assumption College, cette note de recherche suit le parcours migratoire d'une famille bourgeoise de Québec arrivée à Boston en 1890, mettant l'accent sur la question de son intégration (ou non-intégration) à la société américaine sur quatre générations. L'expérience de cette famille, qui n'a rien à voir avec le stéréotype de l'habitant famélique contraint de chercher un travail dans les moulins, montre l'attrait du rêve américain pour les couches plus élevées de la société québécoise des $\mathrm{XIX}^{\mathrm{e}}$ et $\mathrm{XX}^{\mathrm{e}}$ siècles. Elle montre aussi que le processus d'assimilation est très complexe, au moins dans le domaine culturel. En ce qui concerne l'intégration économique, elle est parfaitement réussie. Grâce au travail et à l'entraide de tous les membres, la famille maintient - non sans difficulté - son rang bourgeois malgré la mort du patriarche à 49 ans en 1893. La question de l'intégration culturelle et linguistique est beaucoup plus délicate. Les neuf enfants de la deuxième génération ne partagent ni la même attitude envers l'assimilation ni le même sens d'identité ou d'appartenance ethnique. Le clivage ne se fait pas non plus selon l'âge à l'immigration, malgré la prédiction du père que ses enfants les plus jeunes deviendraient vite de petits Américains. À la troisième et même à la quatrième génération, la situation reste très compliquée. Ce n'est qu’à la fin du $\mathrm{XX}^{\mathrm{e}}$ siècle que la famille se scinde clairement en deux branches, l'une américaine et l'autre québécoise.
All Rights Reserved @ Conseil international d'études canadiennes, 2012
Ce document est protégé par la loi sur le droit d'auteur. L'utilisation des services d'Érudit (y compris la reproduction) est assujettie à sa politique d'utilisation que vous pouvez consulter en ligne.

https://apropos.erudit.org/fr/usagers/politique-dutilisation/ 


\title{
Leslie Choquette
}

\section{Les rêves américain et canadien des Jobin. Une famille bourgeoise de Québec aux États-Unis, 1890-1990}

\section{Résumé}

Basée sur la Collection Jobin, un fonds familial franco-américain conservé à l'Institut français d'Assumption College, cette note de recherche suit le parcours migratoire d'une famille bourgeoise de Québec arrivée à Boston en 1890, mettant l'accent sur la question de son intégration (ou non-intégration) à la société américaine sur quatre générations. L'expérience de cette famille, qui n'a rien à voir avec le stéréotype de l'habitant famélique contraint de chercher un travail dans les moulins, montre l'attrait du rêve américain pour les couches plus élevées de la société québécoise des XIX et XXe siècles. Elle montre aussi que le processus d'assimilation est très complexe, au moins dans le domaine culturel. En ce qui concerne l'intégration économique, elle est parfaitement réussie. Grâce au travail et à l'entraide de tous les membres, la famille maintient - non sans difficulté - son rang bourgeois malgré la mort du patriarche à 49 ans en 1893. La question de l'intégration culturelle et linguistique est beaucoup plus délicate. Les neuf enfants de la deuxième génération ne partagent ni la même attitude envers l'assimilation ni le même sens d'identité ou d'appartenance ethnique. Le clivage ne se fait pas non plus selon l'âge à l'immigration, malgré la prédiction du père que ses enfants les plus jeunes deviendraient vite de petits Américains. À la troisième et même à la quatrième génération, la situation reste très compliquée. Ce n'est qu'à la fin du XX siècle que la famille se scinde clairement en deux branches, l'une américaine et l'autre québécoise.

\begin{abstract}
Based on the Jobin Collection, a Franco-American family archive housed at Assumption College's French Institute, this research note examines the migration of a middle-class family from Quebec City to Boston in 1890, focusing on the question of their integration (or non-integration) into United States society over four generations. The experience of this family, which contrasts sharply with the stereotype of impoverished habitants forced to seek factory work in New England, illustrates the attraction of the American dream for middle-class Quebecers in the $19^{\text {th }}$ and $20^{\text {th }}$ centuries. It also shows that the process of assimilation was very complex, at least in the cultural sphere. Economically, the integration of these immigrants was an unqualified success. Thanks to the hard work and solidarity of all family members, the Jobins held on to their middleclass status despite the death of the patriarch at age 49 in 1893. Their cultural
\end{abstract}


and linguistic integration, on the other hand, was far more complicated. The nine children of the second generation, who immigrated during childhood or adolescence, shared neither the same attitude toward assimilation nor the same ethnic identity. Nor did their choices correspond to their age at immigration, in spite of their father's prediction that his youngest children would quickly become little Americans. For members of the third and even fourth generations, issues of identity remained very complex. It was only at the end of the $20^{\text {th }}$ century that the family divided clearly into two branches, one Anglophone and American, the other Francophone and Québécois.

Cette note de recherche suit le parcours migratoire d'une famille de rêveurs américains, les Jobin, de leur arrivée à Boston en 1890 jusqu'à nos jours. J'ai pu rétablir ce parcours grâce à la Collection Jobin, un fonds familial francoaméricain rassemblé par un membre de la quatrième génération et conservé à l'Institut français d'Assumption College à Worcester, Massachusetts. La collection comprend non seulement de nombreuses lettres - plusieurs centaines - échangées entre divers membres de la famille, mais aussi des ébauches de mémoires d'un membre de la deuxième génération. Ces mémoires prennent la forme de correspondances, car leur auteure, Marie-Eugénie Jobin, les envoyait à ses frères et sœurs pour solliciter leurs réactions, et ils lui répondaient avec des suggestions. Il existe donc de multiples versions de plusieurs épisodes des mémoires de Marie-Eugénie, en anglais ou en français selon leur destinataire.

Voici, selon Marie-Eugénie, les circonstances qui expliquent la décision d'émigrer aux États-Unis et qui n'ont rien à voir avec le stéréotype de l'habitant famélique contraint de chercher un travail temporaire dans les moulins :

Notre père, dont la santé n'avait-jamais été bonne, avait souffert, durant l'hiver précédant, d'une sérieuse pneumonie. Un spécialiste qu'il consulta, lui avoua franchement qu'il ne pouvait vivre même une année s'il ne se rendait pas au Colorado où il pourrait peut-être se guérir. Après un tel verdict que pouvait-faire le père d'une nombreuse famille ( 9 enfants) sans fortune! C'est alors que Dieu vint à son aide en lui envoyant la visite d'un ancien ami du séminaire, M. Arthur Tessier, riche pharmacien, établi à Boston depuis une vingtaine d'années. Il lui conseilla de quitter Québec, cela voulait dire vendre son petit commerce qui lui donnait beaucoup de souci, parce qu'il accordait peut-être trop de crédit aux petits marchands des villages et campagnes [...] Enfin Boston n'était pas le Colorado, mais certainement son climat est moins rigoureux que celui de Québec. Notre père cependant aimait son pays, en outre il n'avait jamais beaucoup voyagé. Il se rendait compte du fait que nous, ses enfants deviendrions des Américains. Tout en hésitant à prendre la seule solution possible sous les circonstances, il se décida donc après avoir réfléchi sérieusement ainsi que d'en avoir causé avec notre chère Maman et ses deux frères, Oncle Louis [1'aîné 
qui avait hérité de la ferme familiale à Saint-Augustin, où il était le maire rouge] et le curé Jobin [Désiré, le cadet]. L'avenir de ses neuf enfants était en jeu. La Nouvelle-Angleterre offrait certainement de plus favorables avantages que Québec, même Montréal. Notre père connaissait bien l'anglais et la comptabilité, ayant suivi deux années de cours supplémentaires dans une école anglaise après le séminaire, il était donc fort bon mathématicien ayant été souvent appelé à démêler les livres de grandes maisons de commerce de même que de certaines banques de Québec, il passa pour un des meilleurs comptables de la ville. Toujours courageux, il se prépara donc à partir avec Théodore et Louis Joseph [ses fils aînés], laissant notre mère, Emma, Eugène et le petit bébé Antoine ( 1 an) chez notre tante Caroline (Mme Mercier) sœur de Maman, qui habitait une maison spacieuse à Lévis. Mon parrain, Joseph Mercier, passait pour riche; propriétaire de deux journaux, Le Quotidien et L'Hebdomadaire, ainsi que d'une librairie et d'un établissement de reliure. (M.-E. Jobin, Notebooks, vol. 3, 22-23)

Dans une version anglaise de la même histoire, Marie-Eugénie ajoute :

Boston, a wonderful and rather aristocratic city, seemed the right place for us all. The schools were free and the best in the country [...] So far the older children had attended the best schools (Ursulines and the Séminaire). Father considered education the main factor in anyone's life, hence the reason we all learned English while living in Quebec. (M.-E. Jobin, Notebooks, vol. 2, 75)

\section{Le chemin des États}

Joseph Jobin part donc pour Boston en août 1890 avec et ses deux fils, d'où il envoie les détails de leur établissement dans deux lettres fascinantes adressées à sa fille Anne-Marie. Dès le 30 août, les garçons ont déjà trouvé un travail dans les grands magasins, en passant par une agence qui prend leur première semaine de paie, $3 \$$ pour commencer. « Notre avenir est maintenant assuré dit-il, il y aura de l'argent en masse à gagner pour tous - toi comme écrivain à la maison, Nini [Marie-Eugénie, qui avait 13 ans] comme apprentie modiste de chapeaux chez une vraie Dame. Elle pourra gagner $1.50 \$$ en faisant son apprentissage et au bout d'une année c'est rien autre chose qu'un salaire de $12 \$$ à $15.00 \$$ par semaine» (J. Jobin, Lettre du 30 août 1890).

Joseph aime beaucoup Boston qui semble incarner pour lui le rêve américain. Il écrit : « On ne peut jamais se faire une idée de la grandeur \& de la beauté de la ville de Boston aussi bien que des affaires qui s'y font » (J. Jobin, Lettre du 30 août 1890). Il conclut :

Je suis maintenant content du mouvement et je voudrais bien avoir fait ce mouvement depuis des années [...] Vous allez en bénéficier. 
Vous allez voir la différence qu'il y a de vivre dans un centre comme celui-ci, et de rester à croupir dans un trou où tout le monde à part une vingtaine sont quêteux. Ce sont des millionnaires ici qui sortent leur argent en font bénéficier toute la population tout en augmentant leurs capitaux. (J. Jobin, Lettre du 30 août 1890)

Ce n'est qu'après coup que Joseph aborde la question de la langue : «P.S. On entend jamais parler français. C'est toujours anglais, il y a de toutes les nationalités dans la ville. Français, Écossais, Chinois, Italiens, Allemands, Sauvages, etc. etc. Chacun porte son costume sans s'occuper de personne " (J. Jobin, Lettre du 30 août 1890).

Quand il écrit une deuxième fois le 17 septembre, Joseph a lui aussi trouvé un emploi, dans les bureaux d'un grand magasin, Jordan Marsh and Company. Comme les garçons, il espère trouver un travail supplémentaire la nuit, mais il a déjà écrit à sa femme de le rejoindre avec les autres enfants par le train du 28 septembre. Hélas, Nini a d'autres idées. Elle écrit :

When I realized that my parents' project of coming to Boston was a serious one to materialize quite soon I cried and cried whole days and whole nights. One day Uncle Louis came to help Father in selling his business, he happened to see me crying \& $[\ldots]$ turning to Mother he said, "What is the matter with that child?" "Oh," said Mother, "she does not want to go to Boston." "Well," Uncle answered, "Why should she go to Boston? There is no need of her being with you, you have enough of eight to attend to. She can remain here as a boarder in the convent, I shall look after her." (M.-E. Jobin, Notebooks, vol. 2, 10-11)

D'où le commentaire de Joseph dans sa deuxième lettre, où il souhaite que Nini vienne avec les autres pour acquérir ce qu'il appelle « des connaissances plus précieuses que celles du couvent ». «Enfin, écrit-il, puisque c'est arrangé, laissons faire [...] Elle aura besoin dans tous les cas de cultiver son anglais. Ici on ne parle pas français. C'est très rare à moins de rencontrer quelques Canadiens que l'on connaît [...] Si tu la vois dis-lui cela, de cultiver son anglais. Je pense bien que dans 5 ou 6 ans d'ici Eugène, Marguerite-Marie et Jeanne ainsi que Gustave [-Antoine] seront plus Anglais que Canadiens » (J. Jobin, Lettre du 17 septembre 1890). Marie-Eugénie a fini par rejoindre sa famille à Boston deux ans plus tard, un an avant le décès de son père de la tuberculose en 1893.

\section{Le rêve réalisé}

Les écrits de la deuxième génération des Jobin, celle de Marie-Eugénie, permettent d'explorer l'importance et les limites de l'intégration de cette famille immigrante à la société américaine. En ce qui concerne l'intégration économique, elle est parfaitement réussie. Grâce au travail et à l'entraide de 
tous les membres, la famille maintient son rang bourgeois malgré la mort du patriarche à 49 ans. Cela ne veut pas dire que le chemin est facile. L'aîné, Théodore, qui a des ambitions artistiques, continue à travailler aux grands magasins, mais comme étalagiste. Sa situation est toujours précaire en 1908, quand sa femme écrit à sa belle-sœur, Anne-Marie Jobin :

[T] his week he is working decorating Mecanic Hall [Mechanics' Hall à Worcester] and there is but little in that, but after Saturday, why he has really nothing in view, he is going around everywhere to try to do wax figures but you don't know how I wish he had a regular position because there is nothing like knowing just what you have to do with. He has not seemed too very fortunate in things he has applied for there have been 3 different places he tried for but did not get. (Thibodeau, vol. 2, 401-403)

Théodore trouve finalement un poste permanent au grand magasin Emerson's à Boston. Sa fille Estelle, qui a quatre ans au moment de la lettre, va faire ses études à Harvard University, devenir institutrice et épouser un avocat, Ralph Thibodeau, avec qui elle aura deux fils, Théodore qui sera dentiste et Philippe qui deviendra prêtre ${ }^{1}$.

Le deuxième fils, Louis-Joseph, reste lui aussi dans le commerce, devenant le propriétaire d'une librairie, Schoenhof's Foreign Books, qui existe toujours à Cambridge. Anne-Marie fait un mariage malheureux, divorce et se consacre à l'immobilier avec beaucoup de succès. Elle possédera même sa propre agence dans le Rhode Island. Marie-Eugénie, dès son arrivée à Boston, suit un cours de couture qui coûte $80 \$$ à la famille. Elle travaille ensuite comme couturière puis comme professeure de dessin de mode à la Boston Trade School.

Quant aux enfants de Joseph qui font tous leurs études aux États-Unis : Eugène achètera une ferme dans le Rhode Island où il fera l'élevage de volailles tout en occupant un poste de facteur; Emma et Marguerite épouseront des hommes d'affaires; Jeanne deviendra secrétaire après avoir suivi un cours commercial; et le bébé, Antoine, obtiendra un diplôme de Harvard et deviendra professeur à l'Université du Michigan.

\section{L'énigme de l'appartenance}

La question de l'intégration culturelle et linguistique est pourtant beaucoup plus complexe. À lire la correspondance, on se rend compte que les neuf enfants de Joseph Jobin ne partagent ni la même attitude envers l'assimilation ni le même sens d'identité ou d'appartenance ethnique. On voit aussi que le clivage ne se fait pas selon l'âge à l'immigration, malgré la prédiction de Joseph Jobin que ses enfants les plus jeunes deviendraient vite de petits Américains. 
C'est chez Anne-Marie, la troisième enfant, âgée de quinze ans en 1890, que l'assimilation est la plus complète. Marie-Eugénie fait un portrait de sa sœur en 1930 :

She acquired the English language better and quicker than all the other members of that large family. Through a devoted admirer [sans doute son futur mari, Paul Davies], she became acquainted with the best English authors, Shakespeare, Scott, Thackeray, Dickens and many others. She read classics, hence her culture. It is to be regretted however that she never read French a great deal, although her brother [elle habitait chez Eugène après son divorce] brought home a large number of French books by the best authors. She remained long after her marriage [c'est-à-dire son divorce] a staunch admirer of the AngloSaxon element. Her friends are almost all Americans, and she herself a $100 \%$ American, preferring U.S. to Canada. (M.-E. Jobin, Notebooks, vol. 2, 97-98)

Comme Anne-Marie, Théodore choisit une conjointe qui n'est pas francophone. Sa femme, Emily Peterson, est une immigrante danoise, une luthérienne qui s'intéresse à la science chrétienne. Elle correspond avec les Jobin en anglais, même si elle ajoute dans une lettre à Marie-Eugénie, écrite en 1909: "Tell mother 'I can read French very nicely even your hastiest note', Ninie" (Thibodeau, vol. 2, 394). La première langue de leur fille Estelle est le danois, mais tout change avec la mort prématurée d'Emily de la tuberculose en 1910 et l'adoption de fait d'Estelle par sa grand-mère Jobin. Estelle fera carrière dans l'enseignement du français tout en épousant un Américain d'ascendance acadienne, Ralph Thibodeau de Old Town, Maine. Si sa correspondance avec Ralph est entièrement en anglais, elle enverra ses deux fils, Teddy et Philippe, à une école paroissiale bilingue à Providence (Saint-Charles, tenue par les religieuses de Jésus-Marie). Il est vrai que Teddy, âgé de six ans en 1948, s'adresse à Santa Claus en anglais, écrivant "Please may I have ice skates? Please may I have wagon and a fire engine ladder truck and a Mickey Mouse wrist watch?" (Thibodeau, vol. 2, 283). Pourtant en 1954, il est capable de remercier un parent canadien de son cadeau de Noël en français :

Chère Cousine Jeanne,

Merci beaucoup du beau livre Pauvre Blaise [un livre d'enfant de la comtesse de Ségur]. J'aime beaucoup la lecture, et je lis le français maintenant assez facilement. J'ai déjà lu Tintin au Congo et Le Sceptre d'Otocar deux des livres de Tintin. Je vais essayer de en trouver d'autres. (Thibodeau, vol. 2, 212-A)

Teddy, aujourd'hui dentiste, habite toujours dans la banlieue de Boston. Son petit frère Philippe, bilingue lui aussi, rentre au Canada comme prêtre du 
diocèse de la Nouvelle-Écosse. Amateur de généalogie et d'histoire familiale, c'est lui le créateur de la Collection Jobin. Pourtant les enfants de Teddy, qui ont tous entre 36 et 43 ans en 2011, sont des anglophones unilingues.

La cousine Jeanne à qui s'adresse Teddy est la fille d'Emma Jobin, la cinquième enfant, qui s'installe à Montréal après son mariage, tout comme la septième enfant, Marguerite. Ces deux filles réussissent parfaitement leur réintégration à la bourgeoisie québécoise, Emma en épousant l'homme d'affaires Hector LaRocque et Marguerite en épousant le financier Édouard Gravel. Ces derniers sont propriétaires d'une belle maison sur la rue McTavish. Il est intéressant de noter qu'Emma et Marguerite ont cinq enfants chacune tandis qu'aucun des sept enfants restés aux États-Unis n'est à l'origine d'une famille nombreuse.

Marie-Eugénie écrit la version française de ses mémoires à l'intention d'Emma et de Marguerite, ses deux sœurs redevenues québécoises. Mais elle choisit de rédiger ses souvenirs d'abord en anglais, langue dans laquelle elle a suivi des cours de création littéraire. Marie-Eugénie, comme ses frères Louis et Antoine, essaie de faire le trait d'union entre ses cultures de naissance et d'adoption. Louis, le propriétaire de Schoenhof's Foreign Books, est membre de la Société historique franco-américaine, secrétaire de la Franco-American Civic League et président du conseil numéro 7 de l'Union Saint-Jean-Baptiste d'Amérique; Antoine, professeur de français à l'Université du Michigan, est l'auteur du livre Visages littéraires du Canada français (A. Jobin, 1941). Mais l'expression la plus parfaite de cette double identité est peut-être le grand spectacle en douze actes créé par Marie-Eugénie à l'occasion du tricentenaire de la ville de Boston en 1930. Intitulé France in the New World, elle le décrit comme suit dans le programme :

This Pageant, France in the New World, will relate to you by means of scenes and tableaux, the important role that France played in the development of the civilization of this continent [...] We, FrancoAmericans, proud descendants of these valiant pioneers, now belong to the most powerful and richest nation of the Universe, and the line that attaches us to this soil is so strong that centuries cannot break the chain that makes us true Americans. (M.-E. Jobin, France in the New World, 4)

\section{Conclusion}

L'expérience de la famille Jobin montre donc à la fois l'attrait du rêve américain pour la couche moyenne de la société québécoise au tournant du $\mathrm{XX}^{\mathrm{e}}$ siècle et la complexité du processus d'assimilation, au moins en ce qui concerne le domaine culturel. Dès la deuxième génération, on est confronté à un éventail de choix culturels allant de l'américanité à $100 \%$ à l'esprit de retour en 
passant par le patriotisme ethnique de gens « loyaux, mais français », pour utiliser l'expression de Mark Paul Richard (2008). À la troisième et même à la quatrième génération, la situation linguistique reste assez floue. Ce n'est qu'à la cinquième génération que les enfants nés aux États-Unis, pour la plupart dans les années 1960 et 1970, se fondent tout à fait dans le fameux creuset américain.

\section{Note}

1. C'est le père Philippe Thibodeau qui rassembla la Collection Jobin et arrangea son transfert à l'Institut français en 1999. En tant que directrice de l'Institut, je tiens à le remercier ici de sa belle vision, son travail acharné et sa grande générosité

\section{Bibliographie}

Jobin, Antoine. Visages littéraires du Canada français. 22 bois originaux de Théodore Jobin. Montréal : Les Éditions du Zodiaque, 1941.

Jobin, Joseph. Lettres à Anne-Marie Jobin, 30 août et 17 septembre 1890. Collection Jobin (Jobin Family Archive), série 1, carton 1, fichier 1. Institut français, Assumption College.

Jobin, Marie-Eugénie. Family History Notebooks, 3 vols. Éd. Philippe Thibodeau. Collection Jobin, série 5, carton 16. Manuscrit originel dans la série 4A, carton 7. Institut français, Assumption College.

- France in the New World: A Pageant of French Civilization in America, 1523-1930. Collection Jobin, série 4A, carton 7, fichier 57. Institut français, Assumption College.

Richard, Mark Paul. Loyal but French: The Negotiation of Identity by FrenchCanadian Descendants in the United States. East Lansing: Michigan State University Press, 2008.

Thibodeau, Philippe, éd. Notes toward a Family History: Photographs, Letters, Memoirs and Other Jottings Related to the Thibodeau Family, 2 vols. Collection Jobin, série 5, carton 13. Institut français, Assumption College. 